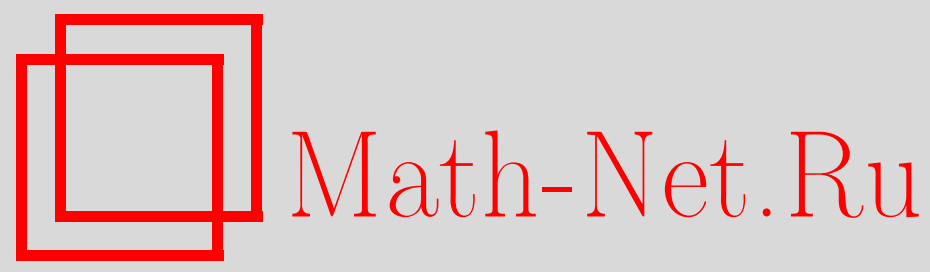

А. С. Леонов, Замечания о полной вариации функций нескольких переменных и многомерном аналоге принципа выбора Хелли, Матем. заметки, 1998, том 63, выпуск 1, 69-80

DOI: https://doi.org/10.4213/mzm1249

Использование Общероссийского математического портала Math-Net.Ru подразумевает, что вы прочитали и согласны с пользовательским соглашением http://www.mathnet.ru/rus/agreement

Параметры загрузки:

IP: 34.229 .108 .108

26 апреля 2023 г., 15:01:57

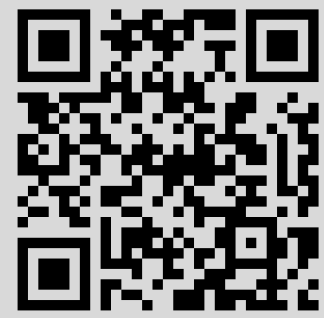




\section{ЗАМЕЧАНИЯ О ПОЛНОЙ ВАРИАЦИИ ФУНКЦИЙ НЕСКОЛЬКИХ ПЕРЕМЕННЫХ И МНОГОМЕРНОМ АНАЛОГЕ ПРИНЦИПА ВЫБОРА ХЕЛЛИ}

\section{А. С. Леонов}

Для класса Харди функций многих переменных с ограниченными вариациями вводится новое понятие полной вариации и устанавливается ряд свойств функций этого класса, аналогичных имеющимся в одномерном случае. В частности, показьвается, что в этом классе справедлив точный аналог принципа выбора Хелли.

Библиографоия: 15 названий.

Известно несколько подходов к конструкции понятия вариации функции нескольких переменных. Таковы, например, вариации Витали, Арцела, Фреше, Тонелли, Хаана, Кронрода-Витушкина, Минлоса и др. (см., например, [1]-[4]). В основном, свойства функций $N$ переменных $(N>1)$ с ограниченной вариацией каждого из этих типов аналогичны свойствам функций с ограниченной вариацией одной переменной. Последние, однако, имеют свойства, вопрос о переносе которых на $N$-мерньй случай требует специального исследования. Это касается, в частности, известного принципа выбора Хелли: из бесконечного семейства функций одной переменной $\{z(x)\}, x \in[a, b]$, которые удовлетворяют условию

$$
|z(a)|+V_{a}^{b}(z) \leqslant C=\text { const, }
$$

можно выделить последовательность, сходящуюся в каждой точке отрезка $[a, b]$ к некоторой функции с конечной вариацией (см. [5, с. 209]).

В данной заметке будет рассматриваться класс Харди $\mathscr{V}_{H}$ функций нескольких переменных с ограниченньми вариациями, введенньй в [6] (см. п. 3). Для функций этого класса в $N$-мерном случае известны утверждения, близкие по сути к принципу выбора Хелли (см., например, [7], [8]). Одно из них приведено ниже в п. 4. Эти утверждения, однако, отличаются от принципа выбора Хелли использованием "сходимости в существенном" вместо поточечной сходимости. Таким образом, и для класса Харди проблема точного аналога принципа выбора Хелли в $N$-мерном случае открыта. Далее будет показано, что такой точный аналог имеется.

В заметке предлагается также иное определение упомянутого класса Харди. Дело в том, что при изучении класса $\mathscr{V}_{H}$ в работах [2], [3], [6] не вводилось единое понятие “вариации функции $N$ переменных", а функция характеризовалась цельм набором вариаций различной размерности. В принципе, такой подход весьма плодотворен особенно для других конструкций вариаций (см. [4], [9]). Вместе с тем, в построенной таким образом в [2], [3] теории функций $N$ переменных с ограниченными вариациями отсутствуют 
аналоги некоторых хорошо известных при $N=1$ утверждений. Например, неравенство для функции одной переменной

$$
|z(x)-z(y)| \leqslant V_{x}^{y}(z) \quad \forall x, y \in[a, b]
$$

не переносится на класс $\mathscr{V}_{H}$ из работ [2], [3]. Оказьвается, что вводя новое определение полной вариации функции $N$ переменных, можно не только охарактеризовать класс $\mathscr{V}_{H}$ как множество функций с ограниченной полной вариацией, но и получить для функций этого класса в $N$-мерном случае ряд дополнительных свойств (например, перенести на $N$-мерньй случай приведенное вьше неравенство и т.п.).

Введение полной вариации, установление дополнительных свойств функций класса Харди, а также $N$-мерного аналога принципа выбора Хелли, важно в связи с рядом приложений. Одно из них - использование функций нескольких переменных с ограниченной вариацией в решении проблемы кусочно-равномерной регуляризации (см. [10, c. 200]) многомерных некорректно поставленных задач.

1. Полная вариация функций нескольких переменных. Пусть $B=B_{a}^{b}=$ $B_{a_{1} \ldots a_{N}}^{b_{1} \ldots b_{N}}=\left\{x \in \mathbb{R}^{N}: a_{i} \leqslant x_{i} \leqslant b_{i}, a_{i}<b_{i}, i=\overline{1, N}\right\}-$ основной брус в $\mathbb{R}^{N}$, в котором определена функция $z(x)=z\left(x_{1}, \ldots, x_{N}\right)$. Введем в $B$ частичную упорядоченность точек $\alpha, \beta \in B$ с помощью конуса $K^{+}=\left\{x \in \mathbb{R}^{N}: x_{i}>0, i=\overline{1, N}\right\}$ :

$$
\begin{aligned}
& \alpha<\beta, \text { если } \beta-\alpha \in K^{+} ; \\
& \alpha \leqslant \beta, \text { если } \beta-\alpha \in \bar{K}^{+} .
\end{aligned}
$$

Будем обозначать как $B_{\alpha}^{\beta}=B_{\alpha_{1} \ldots \alpha_{N}}^{\beta_{1} \ldots \beta_{N}}=\left\{x \in \mathbb{R}^{N}: \alpha_{i}<x_{i} \leqslant \beta_{i}, i=\overline{1, N}\right\}, \alpha<\beta$, (координатные) подбрусы размерности $N$ основного бруса. Если при этом $\alpha_{i}=a_{i}$, то неравенство $\alpha_{i}<x_{i}$ заменяется здесь на $\alpha_{i} \leqslant x_{i}$. Определим с помощью функции $z(x)$ " $N$-мерный квазиобъем" $\sigma_{N}$ бруса $B_{\alpha}^{\beta}$ по правилу:

$$
\sigma_{N}\left(B_{\alpha}^{\beta}\right)=\sum_{\nu_{1}=0}^{1} \cdots \sum_{\nu_{N}=0}^{1}(-1)^{\nu_{1}+\cdots+\nu_{N}} z\left[\beta_{1}+\nu_{1}\left(\alpha_{1}-\beta_{1}\right), \ldots, \beta_{N}+\nu_{N}\left(\alpha_{N}-\beta_{N}\right)\right]
$$

(см. [7], [11], [12]). Например, при $N=2$ получим

$$
\sigma_{2}\left(B_{\alpha}^{\beta}\right)=\sigma_{2}\left(B_{\alpha_{1} \alpha_{2}}^{\beta_{1} \beta_{2}}\right)=z\left(\beta_{1}, \beta_{2}\right)-z\left(\beta_{1}, \alpha_{2}\right)-z\left(\alpha_{1}, \beta_{2}\right)+z\left(\alpha_{1}, \alpha_{2}\right) .
$$

Квазиобъем $\sigma_{N}$ является аддитивной функцией брусов (см. [12, с. 162]). Зададим допустимое разбиение П основного бруса: $\Pi=\left\{B_{j}\right\}_{j=1}^{n}$, в котором $N$-мерные подбрусы $B_{j}$ не пересекаются и $B=B_{1}+\cdots+B_{n}$. Совокупность всех таких разбиений для всевозможных $n$ обозначим как $\mathscr{P}$. Тогда $N$-мерной вариацией (вариацией Витали) функции $z(x)$ в брусе $B$ назьвается величина

$$
V_{N}(z, B)=\sup _{\Pi}\left\{\sum_{j=1}^{n}\left|\sigma_{N}\left(B_{j}\right)\right|: \Pi=\left\{B_{j}\right\}_{j=1}^{n} \in \mathscr{P}\right\}
$$

(см. [1], [3]). Вариация $V_{N}(z, \cdot)$ представляет собой аддитивную функцию $N$-мерных брусов [12]. Нетрудно видеть, что $V_{N}\left(z_{1}+z_{2}, B\right) \leqslant V_{N}\left(z_{1}, B\right)+V_{N}\left(z_{2}, B\right)$ для любых $z_{1}(x), z_{2}(x)$, имеющих в $B$ конечную вариацию. Отметим также равенство

$$
V_{N}(z, B)=\sup _{\Pi}\left\{\sum_{j}\left|\sigma_{N}\left(B_{j}\right)\right|: \Pi=\left\{B_{j}\right\} \in \mathscr{P}^{*}\right\},
$$


где $\mathscr{P}^{*}$ - совокупность всех “специальных допустимых разбиений" $\left\{B_{j}\right\}$ бруса $B$, порождаемых $N$-мерными сетками вида $S=\left\{x_{k_{1} \ldots k_{N}}: k_{1}=1, \ldots, K_{1}, \ldots, k_{N}=1, \ldots, K_{N}\right\}$, с узлами $x_{k_{1} \ldots k_{N}}=\left(x_{1}^{\left(k_{1}\right)}, \ldots, x_{N}^{\left(k_{N}\right)}\right), a_{i}=x_{i}^{(1)}<x_{i}^{(2)}<\cdots<x_{i}^{\left(K_{i}\right)}=b_{i}, i=\overline{1, N}, \mathrm{\text {в }}$ брусе $B$.

Фиксируем далее число $m(1 \leqslant m \leqslant N-1)$ и рассмотрим $m$-мерную координатную грань $B_{i_{1} \ldots i_{m}}$ основного бруса, проходящую через точку $a \in B$ параллельно осям $x_{i_{1}}$, $\ldots, x_{i_{m}}\left(1 \leqslant i_{1}<\cdots<i_{m} \leqslant N\right)$. Для всякой такой грани $B_{i_{1} \ldots i_{m}}$, как для бруса размерности $m$, можно, используя соотношения типа $(1),(2)$, ввести понятие $m$-мерного квазиобъема $\sigma_{m}$ и $m$-мерной вариации $V_{m}$ функции $z$ по $B_{i_{1}} \ldots i_{m}$. Иногда удобно при каждом $m(1 \leqslant m \leqslant N-1)$ занумеровать все $m$-мерные грани вида $B_{i_{1} \ldots i_{m}}$ индексом $l$ $\left(1 \leqslant l \leqslant C_{N}^{m}=N_{m}\right)$ и обозначить их как $B_{l}^{(m)}$, а их совокупность - как $\mathscr{B}_{m}: \mathscr{B}_{m}=$ $\left\{B_{l}^{(m)}\right\}_{l=1}^{N_{m}}$.

ОПРЕДЕЛЕНИЕ 1. Полной вариацией функиии $z$ в брусе $B$ назовем величину

$$
V(z, B)=\sum_{m=1}^{N-1}\left\{\sum_{l=1}^{N_{m}} V_{m}\left(z, B_{l}^{(m)}\right)\right\}+V_{N}(z, B) .
$$

Множество $\mathscr{V}=\mathscr{V}(B)$ всех функций $z(x)$, определенных в $B$ и имеющих там конечную полную вариацию (4), будем называть пространством функций $N$ переменных с ограниченной полной вариачией.

Если, используя введенные обозначения, считать, что $B_{12} \ldots N=B$, то выражение (4) можно записать в виде следующей суммы $2^{N}-1$ слагаемых:

$$
\begin{aligned}
V(z, B) & =\sum_{m=1}^{N}\left\{\sum_{i_{1}=1}^{m} \cdots \sum_{\substack{i_{m}=1 \\
1 \leqslant i_{1}<\cdots<i_{m} \leqslant N}}^{m} V_{m}\left(z, B_{i_{1} \ldots i_{m}}\right)\right\} \\
& =\sum_{m=1}^{N}\left\{\sum_{i_{1}=1}^{N-m+1} \sum_{i_{2}=i_{1}+1}^{N-m+2} \cdots \sum_{i_{m}=i_{m-1}+1}^{N} V_{m}\left(z, B_{i_{1} \ldots i_{m}}\right)\right\} .
\end{aligned}
$$

В качестве примера приведем выражение полной вариации для $N=2$

$$
V(z, B)=V_{1}\left(z, B_{1}^{(1)}\right)+V_{1}\left(z, B_{2}^{(1)}\right)+V_{2}(z, B)
$$

где $V_{1}(z, \cdot)$ - обычная (одномерная) вариация функции $z\left(x_{1}, x_{2}\right)$ по граням $B_{1}^{(1)}=\left\{a_{1} \leqslant\right.$ $\left.x_{1} \leqslant b_{1}, x_{2}=a_{2}\right\}, B_{2}^{(1)}=\left\{x_{1}=a_{1}, a_{2} \leqslant x_{2} \leqslant b_{2}\right\}$ бруса $B=\left[a_{1}, b_{1}\right] \times\left[a_{2}, b_{2}\right] \subset \mathbb{R}^{2}$.

Полную вариацию можно записать и в другой форме. Для этого зададим в брусе $B$ сетку $S$, как это делалось выше. Она порождает для $B$ специальное разбиение $\left\{B^{k_{1} \ldots k_{N}}: k_{i}=1, \ldots, K_{i}-1, i=\overline{1, N}\right\}$ на подбрусы $B^{k_{1} \ldots k_{N}}=\left\{\left(x_{1}, \ldots, x_{N}\right): x_{i}^{\left(k_{i}\right)}<\right.$ $\left.x_{i} \leqslant x_{i}^{\left(k_{i}+1\right)}, i=\overline{1, N}\right\}$. Кроме того, она порождает специальные разбиения $\left\{B^{k_{i_{1}} \ldots k_{i_{m}}}\right\}$ каждой $m$-мерной грани $B_{i_{1} \ldots i_{m}} \in \mathscr{B}_{m}(m=\overline{1, N-1})$ бруса $B$ на подбрусы $B^{k_{i_{1}} \ldots k_{i_{m}}}=$ $\left\{\left(x_{i_{1}}, \ldots, x_{i_{m}}\right): x_{i_{p}}^{\left(k_{i_{p}}\right)}<x_{i_{p}} \leqslant x_{i_{p}}^{\left(k_{i_{p}}+1\right)}, p=\overline{1, m}\right\}$. Все эти разбиения можно единообразно описать, приняв следующее соглашение. Будем формально считать, что при $k_{i}=0$ неравенство $x_{i}^{(0)}<x_{i} \leqslant x_{i}^{(1)}$ в определении подбруса $B^{k_{1} \ldots k_{N}}$ означает, что 
$x_{i}=x_{i}^{(1)}=a_{i}$. Тогда $m$-мерные подбрусы $B^{k_{i_{1}} \ldots k_{i m}}$ формально представляют собой подбрусы вида $B^{k_{1} \ldots k_{N}}$, у которых в наборе $\left(k_{1}, \ldots, k_{N}\right)$ индексы $k_{i_{1}}, \ldots, k_{i_{m}}$ отличны от 0 , а остальные - равны 0. Приняв это соглашение, будем иногда в дальнейшем обозначать квазиобъемы различных размерностей $m \sigma_{m}\left(B^{k_{i_{1}} \ldots k_{i_{m}}}\right)$ как $\sigma\left(B^{k_{1} \ldots k_{N}}\right)$.

Введем для сетки $S$ величину

$$
\begin{aligned}
\omega(z, S) & =\sum_{k_{1}=0}^{K_{1}-1} \cdots \sum_{\substack{k_{N}=0 \\
k_{1}+\cdots+k_{N}>0}}^{K_{N}-1}\left|\sigma\left(B^{k_{1} \ldots k_{N}}\right)\right| \\
& =\sum_{m=1}^{N}\left\{\sum_{i_{1}=1}^{m} \cdots \sum_{i_{m}=1}^{m}\left[\sum_{k_{i_{1}}=1}^{K_{1}<\cdots<i_{m} \leqslant N} \ldots \sum_{k_{i_{m}}=1}^{K_{i_{1}}-1}\left|\sigma_{m}\left(B^{k_{i_{1}} \ldots k_{i_{m}}}\right)\right|\right]\right\} \\
& =\sum_{m=1}^{N}\left\{\sum_{i_{1}=1}^{N-m+1} \sum_{i_{2}=i_{1}+1}^{N-m+2} \cdots \sum_{i_{m}=i_{m-1}+1}^{N}\left[\sum_{k_{i_{1}}=1}^{K_{i_{1}}-1} \ldots \sum_{k_{i_{m}}=1}^{K_{i_{1}}-1} \mid \sigma_{m}\left(B^{\left.k_{i_{1}} \ldots k_{i_{m}}\right) \mid}\right]\right\} .\right.
\end{aligned}
$$

В частности, при $N=2$ для сетки $S=\left\{x_{k l}: k=1, \ldots, K, \ldots, l=1, \ldots, L\right\}$ в брусе $B \subset \mathbb{R}^{2}$ выражение (6) записывается в виде

$$
\begin{aligned}
\omega(z, S)= & \sum_{k=0}^{K-1} \sum_{l=0}^{L-1}\left|\sigma\left(B^{k l}\right)\right|=\sum_{k=1}^{K-1}\left|\sigma\left(B^{k 0}\right)\right|+\sum_{l=1}^{L-1}\left|\sigma\left(B^{0 l}\right)\right|+\sum_{k=1}^{K-1} \sum_{l=1}^{L-1}\left|\sigma\left(B^{k l}\right)\right| \\
= & \sum_{k=1}^{K-1}\left|z\left(x_{k+1,1}\right)-z\left(x_{k 1}\right)\right|+\sum_{l=1}^{L-1}\left|z\left(x_{1, l+1}\right)-z\left(x_{1 l}\right)\right| \\
& +\sum_{k=1}^{K-1} \sum_{l=1}^{L-1}\left|z\left(x_{k+1, l+1}\right)-z\left(x_{k+1, l}\right)-z\left(x_{k, l+1}\right)+z\left(x_{k l}\right)\right| .
\end{aligned}
$$

Пусть $\mathscr{S}$ - множество всевозможных сеток $S$ в брусе $B \subset \mathbb{R}^{N}$. Тогда справедлива

Теорема 1. Для всякой функиии $z \in \mathscr{V}(B)$

$$
V(z, B)=\sup _{S}\{\omega(z, S): S \in \mathscr{S}\}
$$

ДокАЗАТЕльство. Зададим произвольное $\varepsilon>0$. Для него найдется столь "мелкое" разбиение $\left\{B^{k_{1} \ldots k_{N}}\right\} \in \mathscr{P}^{*}(B)$ бруса $B$, которое в силу равенства типа (3) для вариации $V_{m}(z, \cdot)$ обеспечивает на соответствующих разбиениях $\left\{B^{k_{i_{1}} \ldots k_{i_{m}}}\right\}$ всех $m$-мерных граней $B_{i_{1} \ldots i_{m}} \in \mathscr{B}_{m}\left(1 \leqslant i_{1}<\ldots<i_{m} \leqslant N\right)$ произвольной размерности $m(1 \leqslant m \leqslant N)$ выполнение неравенств

$$
V_{m}\left(z, B_{i_{1} \ldots i_{m}}\right)-\frac{\varepsilon}{2^{N}-1} \leqslant \sum_{k_{i_{1}}=1}^{K_{i_{1}}-1} \cdots \sum_{k_{i_{m}}=1}^{K_{i_{m}}-1}\left|\sigma_{m}\left(B^{k_{i_{1}} \ldots k_{i_{m}}}\right)\right| .
$$

Суммируя все такие неравенства по $m$ и по $i_{1}, \ldots, i_{m}\left(1 \leqslant i_{1}<\cdots<i_{m} \leqslant N\right)$, с учетом (5) и (6) получим $V(z, B)-\varepsilon \leqslant \omega(z, S) \leqslant V(z, B)$, что и доказывает теорему. 


\section{2. Некоторые свойства функций с ограниченной полной вариацией.}

ТЕОрема 2. Для всякой функиии $z(x)$, определенной в $N$-мерном брусе $B=B_{a}^{b}$, и для любого разбиения $\left\{B^{k_{1} \ldots k_{N}}: k_{i}=0, \ldots, K_{i}-1, i=\overline{1, N}\right\}$ этого бруса справедливо равенство

$$
\begin{aligned}
z(b)-z(a) & =\sum_{k_{1}=0}^{K_{1}-1} \cdots \sum_{\substack{k_{N}=0 \\
k_{1}+\cdots+k_{N}>0}}^{K_{N}-1} \sigma\left(B^{k_{1} \ldots k_{N}}\right) \\
& =\sum_{m=1}^{N}\left\{\sum_{i_{1}=1}^{N-m+1} \sum_{i_{2}=i_{1}+1}^{N-m+2} \cdots \sum_{i_{m}=i_{m-1}+1}^{N}\left[\sum_{k_{i_{1}}=1}^{K_{i_{1}}-1} \cdots \sum_{k_{i_{m}}=1}^{K_{i_{m}}-1} \sigma_{m}\left(B^{k_{i_{1}} \ldots k_{i_{m}}}\right)\right]\right\} .
\end{aligned}
$$

ДоКАЗАТЕЛЬСТво. Вследствие аддитивности квазиобъемов $\sigma_{m}$ верна формула

$$
\sigma_{m}\left(B_{i_{1} \ldots i_{m}}\right)=\sum_{k_{i_{1}}=1}^{K_{i_{1}}-1} \cdots \sum_{k_{i_{m}}=1}^{K_{i_{m}}-1} \sigma_{m}\left(B^{k_{i_{1}} \ldots k_{i_{m}}}\right) .
$$

Поэтому для доказательства равенства (7) достаточно убедиться в том, что

$$
\Sigma \equiv \sum_{m=1}^{N}\left\{\sum_{i_{1}=1}^{N-m+1} \sum_{i_{2}=i_{1}+1}^{N-m+2} \cdots \sum_{i_{m}=i_{m-1}+1}^{N} \sigma_{m}\left(B_{i_{1} \ldots i_{m}}\right)\right\}=z(b)-z(a) .
$$

Согласно формуле типа (1) величины $\sigma_{m}\left(B_{i_{1} \ldots i_{m}}\right)$ линейно выражаются через значения функции $z(x)$ в вершинах бруса $B_{i_{1} \ldots i_{m}} \in \mathscr{B}_{m}$. Поэтому сумма $\Sigma$ есть линейная комбинация всех значений $z(x)$ в вершинах основного бруса $B_{a}^{b}$. Если обозначить эти вершины как $\gamma_{i_{1} \ldots i_{m}}=\left(a_{1}, \ldots, a_{i_{1}-1}, b_{i_{1}}, a_{i_{1}+1}, \ldots, a_{i_{m}-1}, b_{i_{m}}, a_{i_{m}+1}, \ldots a_{N}\right), m=\overline{1, N}$, $1 \leqslant i_{1}<\cdots<i_{m} \leqslant N$, то

$$
\begin{aligned}
\Sigma= & A_{0} z(a)+\sum_{i_{1}=1}^{N} A_{i_{1}} z\left(\gamma_{i_{1}}\right)+\sum_{i_{1}=1}^{N-1} \sum_{i_{2}=i_{1}+1}^{N} A_{i_{1} i_{2}} z\left(\gamma_{i_{1} i_{2}}\right)+\cdots \\
& +\sum_{i_{1}=1}^{N-m+1} \sum_{i_{2}=i_{1}+1}^{N-m+2} \cdots \sum_{i_{m}=i_{m-1}+1}^{N} A_{i_{1} \ldots i_{m}} z\left(\gamma_{i_{1} \ldots i_{m}}\right)+\cdots+A_{12 \ldots N} z(b) .
\end{aligned}
$$

Так как точка $a$ принадлежит всем граням из множества $\left\{\mathscr{B}_{m}\right\}_{m=1}^{N}$, учитьвая, что число граней, содержащихся в $\mathscr{B}_{m}$, равно $C_{N}^{m}$ и что величина $z(a)$ входит в выражение для $\sigma_{m}$ со знаком $(-1)^{m}$, получим

$$
A_{0}=(-1)^{1} C_{N}^{1}+(-1)^{2} C_{N}^{2}+\cdots+(-1)^{N} C_{N}^{N}=(1-1)^{N}-1=-1 .
$$

Точка $b$ принадлежит единственной “грани" вида $B_{i_{1}} \ldots i_{m}$, а именно основному брусу $B=B_{12} \ldots N$, а число $z(b)$ входит в формулу $(1)$ для $\sigma_{N}(B)$ со зна́ком “+”. Поэтому $A_{12 \ldots N}=1$. Принимая во внимание, что число всех возможньг $r$-мерных граней из множества $\mathscr{B}_{r}(m \leqslant r \leqslant N)$, содержащих фиксированную вершину $\gamma_{i_{1} \ldots i_{m}}$, равно $C_{N-m}^{r-m}$, и учитьвая знак $(-1)^{r-m}$ при $z\left(\gamma_{i_{1} \ldots i_{m}}\right)$ в формуле (1) для квазиобъемов этих граней, найдем также

$$
A_{i_{1} \ldots i_{m}}=(-1)^{0} C_{N-m}^{0}+(-1)^{1} C_{N-m}^{1}+\cdots+(-1)^{N-m} C_{N-m}^{N-m}=(1-1)^{N-m}=0 .
$$

Таким образом, из (9), (10) следует, что $\Sigma=z(b)-z(a)$. 
ЗАмЕчаниЕ. Формулы, аналогичные (7), (8), верны и в случае $a \leqslant b$. Например, если $b=\left(a_{1}, \ldots, a_{m}, b_{m+1}, \ldots, b_{N}\right)$, то

$$
z(b)-z(a)=\sum_{k=1}^{N-m}\left\{\sum_{\substack{i_{1} \\ m+1 \leqslant i_{1}<\cdots<i_{k} \leqslant N}} \cdots \sum_{\substack{i_{k} \\ m}} \sigma_{k}\left(B_{i_{1} \ldots i_{k}}\right)\right\} .
$$

Доказательство этой формулы сходно с приведенньм вьше.

В частном случае $N=2$ формула (7) имеет вид

$$
\begin{aligned}
& z\left(b_{1}, b_{2}\right)-z\left(a_{1}, a_{2}\right)=\sum_{k=1}^{K-1}\left[z\left(x_{k+1,1}\right)-z\left(x_{k 1}\right)\right] \\
& +\sum_{l=1}^{L-1}\left[z\left(x_{1, l+1}\right)-z\left(x_{1 l}\right)\right]+\sum_{k=1}^{K-1} \sum_{l=1}^{L-1}\left[z\left(x_{k+1, l+1}\right)-z\left(x_{k+1, l}\right)-z\left(x_{k, l+1}\right)+z\left(x_{k l}\right)\right] .
\end{aligned}
$$

Из теоремы 1 , формулы (6) и равенств вида $(7),(8),(11)$, доказанных в теореме 2 , вытекает ряд следствий, выражающих свойства функций класса $\mathscr{V}(B)$.

СлЕДСТВИЕ 1. Для любой функиии $z(x) \in \mathscr{V}(B)$ выполнены неравенства

$$
\begin{gathered}
|z(x)-z(a)| \leqslant V\left(z, B_{a}^{x}\right) \quad \forall x \in B, \quad x \geqslant a, \\
|z(x)| \leqslant|z(a)|+V\left(z, B_{a}^{x}\right) \leqslant|z(a)|+V(z, B) \quad \forall x \in B .
\end{gathered}
$$

Аналогичные неравенства при $N=1$ хорошо известны [5, с. 203].

СЛЕДСТВИЕ 2. Пространство $\mathscr{V}(B)$ мохнно нормировать, полагая $\|z\|_{V}=$ $|z(a)|+V(z, B)$. Полученное нормированное пространство будет банаховым.

Доказательство этого утверждения проводится почти дословно, как в одномерном случае. При этом существенно используется неравенство (12) и выражение для полной вариации из теоремы 1 (cp. [13, с. 159]).

Отметим, что как следует из (4), эквивалентная норма в $\mathscr{V}(B)$ может быть задана равенством

$$
\|z\|=|z(a)|+\max _{1 \leqslant m \leqslant N}\left\{\max _{B_{l}^{(m)} \in \mathscr{B}_{m}} V_{m}\left(z, B_{l}^{(m)}\right\} .\right.
$$

3. Полностью монотонные функции и некоторые их свойства. Рассмотрим специальный класс $\mathscr{M}(B)$ функций, определенных в $B$.

ОПРЕДЕЛЕНИЕ 2. Будем считать, что $z(x) \in \mathscr{M}(B)$, если для любого бруса $B_{x}^{y}$, $B_{x}^{y} \subset B, x \leqslant y$, произвольной размерности $m(1 \leqslant m \leqslant N)$ квазиобъем $\sigma_{m}\left(B_{x}^{y}\right)$, порожденный функцией $z$, неотрицателен.

Из равенства вида (8), записанного для бруса $B_{x}^{y}$ вместо $B_{a}^{b}$, ясно, что всякая функция $z(x) \in \mathscr{M}(B)$ монотонна (не убывает) относительно конуса $\bar{K}^{+}: z(y) \geqslant z(x)$ для любых $x, y \in B, y \geqslant x$. Из (6), (7) и теоремы 1 следует равенство $V(z, B)=z(b)-z(a)$, и поэтому $\mathscr{M}(B) \subset \mathscr{V}(B)$. При $N=2$ включение $z(x) \in \mathscr{M}(B)$ означает, что

$$
\begin{gathered}
z\left(y_{1}, x_{2}\right)-z\left(x_{1}, x_{2}\right) \geqslant 0, \quad z\left(x_{1}, y_{2}\right)-z\left(x_{1}, x_{2}\right) \geqslant 0 \\
z\left(y_{1}, y_{2}\right)-z\left(x_{1}, y_{2}\right)-z\left(y_{1}, x_{2}\right)+z\left(x_{1}, x_{2}\right) \geqslant 0
\end{gathered}
$$

для любых $\left(x_{1}, x_{2}\right),\left(y_{1}, y_{2}\right) \in B, x_{1} \leqslant y_{1}, x_{2} \leqslant y_{2}$ (см. [6]).

Функции класса $\mathscr{M}(B)$ рассматривались в [2], [14] под названием "квазимонотонные", или “полностью монотонные". Одно из свойств этих функций дает установленная в [14] 
Лемма 1. Полностью монотонные функиии непрерывны в В всюду кроме, быть может, точек разрыва, которые лежат на не более чем счетном, множестве координатных гиперплоскостей размерности $N-1$.

Полностью монотонные функции играют в теории функций $N$ переменных с ограниченной полной вариацией ту же роль, что и монотонные функции для функций одной переменной с ограниченной вариацией. Это, в частности, подтверждается следующей теоремой.

Tеорема 3. Eсли $z(x) \in \mathscr{V}(B)$, то функиии $\pi(x)=V\left(z, B_{a}^{x}\right), \mu(x)=\pi(x)-z(x)$ полностью монотонные в $B$.

ДокАЗАТЕЛЬСТво. Возьмем произвольньй брус $B_{x}^{y} \subset B$ размерности $m(1 \leqslant m \leqslant N)$ и установим, что его квазиобъем $\sigma_{m}\left(\pi, B_{x}^{y}\right)$, порождаемый функцией $\pi$, неотрицателен. Для определенности будем считать, сначала, что брус имеет вид $B_{x}^{y}=B_{x^{\prime} x^{\prime \prime}}^{y^{\prime}}$, где $x^{\prime}=\left(x_{1}, \ldots, x_{m}\right), x^{\prime \prime}=\left(x_{m+1}, \ldots, x_{N}\right), y^{\prime}=\left(y_{1}, \ldots, y_{m}\right)$ и $y^{\prime}>x^{\prime}>a^{\prime}=\left(a_{1}, \ldots, a_{m}\right)$, $x^{\prime \prime}>a^{\prime \prime}=\left(a_{m+1}, \ldots, a_{N}\right)$. Тогда из формулы (1) для квазиобъемов и формулы (5) для полной вариации функции $z$ по $N$-мерному брусу следует равенство

$$
\begin{aligned}
\sigma_{m}\left(\pi, B_{x^{\prime} x^{\prime \prime}}^{y^{\prime} x^{\prime \prime}}\right) & =\sum_{\nu_{1}=0}^{1} \cdots \sum_{\nu_{m}=0}^{1}(-1)^{\nu_{1}+\cdots+\nu_{m}} \pi\left[y^{\prime}+\nu\left(x^{\prime}-y^{\prime}\right), x^{\prime \prime}\right] \\
& =\sum_{\nu_{1}=0}^{1} \cdots \sum_{\nu_{m}=0}^{1}(-1)^{\nu_{1}+\cdots+\nu_{m}} V\left(z, B_{a^{\prime} a^{\prime \prime}}^{d(\nu) x^{\prime \prime}}\right) \\
& =\sum_{\nu_{1}=0}^{1} \cdots \sum_{\nu_{m}=0}^{1}(-1)^{\nu_{1}+\cdots+\nu_{m}}\left\{\sum_{k=1}^{N} \sum_{\substack{i_{1} \\
1 \leqslant i_{1}<\cdots<i_{k} \leqslant N}} \cdots \sum_{i_{k}} V_{k}\left[z,\left(B_{a^{\prime} a^{\prime \prime}}^{d(\nu) x^{\prime \prime}}\right)_{i_{1} \cdots i_{k}}\right]\right\} \\
& =\sum_{k=1}^{N} \sum_{\substack{i_{1} \\
1 \leqslant i_{1}<\cdots<i_{k} \leqslant N}} \ldots \sum_{i_{i}}\left\{\sum_{\nu_{1}=0}^{1}(-1)^{\nu_{1}+\cdots+\nu_{m}} V_{k}\left[z,\left(B_{a}^{d(\nu) x^{\prime \prime}}\right)_{i_{1} \ldots i_{k}}\right]\right\} .
\end{aligned}
$$

Здесь $d(\nu)=y^{\prime}+\nu\left(x^{\prime}-y^{\prime}\right)=\left(y_{1}+\nu_{1}\left(x_{1}-y_{1}\right), \ldots, y_{m}+\nu_{m}\left(x_{m}-y_{m}\right)\right), \mathrm{a}\left(B_{a}^{d(\nu) x^{\prime \prime}}\right)_{i_{1} \ldots i_{k}-}$ $k$-мерная грань $N$-мерного бруса $B_{a}^{d(\nu) x^{\prime \prime}}$, проходящая через точку $a$ параллельно осям с номерами $i_{1}, \ldots, i_{k}$. Обозначим для краткости

$$
S_{i_{1} \ldots i_{k}} \equiv \sum_{\nu_{1}=0}^{1} \cdots \sum_{\nu_{m}=0}^{1}(-1)^{\nu_{1}+\cdots+\nu_{m}} V_{k}\left[z,\left(B_{a}^{d(\nu) x^{\prime \prime}}\right)_{i_{1} \ldots i_{k}}\right] .
$$

Вычислим величины $S_{i_{1} \ldots i_{k}}$ при $k<m$. В этом случае для точек бруса $\left(B_{a}^{d(\nu) x^{\prime \prime}}\right)_{i_{1} \ldots i_{k}}$ хотя бы одна из первых $m$ координат постоянна и совпадает с соответствующей координатой точки $a$. Пусть эта координата имеет номер $j(1 \leqslant j \leqslant m)$. Тогда она не зависит от $\nu_{j}$, и поэтому

$$
\begin{aligned}
S_{i_{1} \ldots i_{k}}= & \sum_{\nu_{1}=0}^{1} \cdots \sum_{\nu_{j-1}=0}^{1} \sum_{\nu_{j+1}=0}^{1} \cdots \sum_{\nu_{m}=0}^{1}(-1)^{\nu_{1}+\cdots+\nu_{j-1}+\nu_{j+1}+\cdots+\nu_{m}} \\
& \times V_{k}\left[z,\left(B_{a}^{d(\nu) x^{\prime \prime}}\right)_{i_{1} \ldots i_{k}}\right] \sum_{\nu_{j}=0}^{1}(-1)^{\nu_{j}}=0 \quad \forall i_{1}, \ldots, i_{k}: \quad 1 \leqslant i_{1}<\cdots<i_{k} \leqslant N .
\end{aligned}
$$


Вследствие этого сумма (13) преобразуется к виду

$$
\begin{aligned}
& \sigma_{m}\left(\pi, B_{x^{\prime} x^{\prime \prime}}^{y^{\prime} x^{\prime \prime}}\right)=\sum_{k=m}^{N}\left(\sum_{\substack{i_{1} \\
1 \leqslant i_{1}<\cdots<i_{k} \leqslant N}} \cdots \sum_{i_{k}} S_{i_{1} \ldots i_{k}}\right) \\
& =\sum_{\substack{i_{1} \\
1 \leqslant i_{1}<\cdots<i_{m} \leqslant N}} \cdots \sum_{i_{i} \ldots i_{m}}+\sum_{i_{1}} \cdots \sum_{i_{m}} \sum_{i_{m+1}} S_{i_{1} \ldots i_{m} i_{m+1}}+\cdots+S_{12 \ldots N} \\
& \quad \equiv \Sigma_{m}+\Sigma_{m+1}+\cdots+\Sigma_{N} .
\end{aligned}
$$

Вычислим сумму $\Sigma_{m}$. Если рассмотреть ее слагаемое $S_{12} \ldots m$, т.е. положить $i_{1}=1$, $i_{2}=2, \ldots, i_{m}=m$, то $\left(B_{a}^{d(\nu) x^{\prime \prime}}\right)_{i_{1} \ldots i_{m}}=B_{a^{\prime} a^{\prime \prime}}^{d(\nu) a^{\prime \prime}}$. Введем оператор $\Delta_{i}(1 \leqslant i \leqslant m)$, преобразуюший брусы $B_{c a^{\prime \prime}}^{d a^{\prime \prime}}$, у которых $c=\left(c_{1}, \ldots, c_{m}\right)<d=\left(d_{1}, \ldots, d_{m}\right)$, по правилу

$$
\Delta_{i} B_{c a^{\prime \prime}}^{d a^{\prime \prime}}=B_{c_{1} \ldots c_{i-1} x_{i} c_{i+1} \ldots c_{m} a^{\prime \prime}}^{d_{1} \ldots d_{i-1} y_{i} d_{i+1} \ldots d_{m} a^{\prime \prime}}=B_{c a^{\prime \prime}}^{d_{1} \ldots d_{i-1} y_{i} d_{i+1} \ldots d_{m} a^{\prime \prime}} \backslash B_{c a^{\prime \prime}}^{d_{1} \ldots d_{i-1} x_{i} d_{i+1} \ldots d_{m} a^{\prime \prime}} .
$$

Тогда из представления $B_{x^{\prime} a^{\prime \prime}}^{y^{\prime} a^{\prime \prime}}=\Delta_{1}\left(\Delta_{2}\left(\ldots\left(\Delta_{m} B_{a^{\prime} a^{\prime \prime}}^{d(\nu) a^{\prime \prime}}\right) \ldots\right)\right)$ и из аддитивности вариации $V_{m}$ функции $z$ по грани $B_{a^{\prime} a^{\prime \prime}}^{y^{\prime} a^{\prime \prime}}$ получим

$$
\begin{aligned}
S_{i_{1} \ldots i_{m}}=S_{12 \ldots m} & =\sum_{\nu_{1}=0}^{1} \cdots \sum_{\nu_{m}=0}^{1}(-1)^{\nu_{1}+\cdots+\nu_{m}} V_{m}\left[z, B_{a^{\prime} a^{\prime \prime}}^{d(\nu) a^{\prime \prime}}\right] \\
& =V_{m}\left[z, \Delta_{1}\left(\Delta_{2}\left(\ldots\left(\Delta_{m} B_{a^{\prime} a^{\prime \prime}}^{d(\nu) a^{\prime \prime}}\right) \ldots\right)\right)\right]=V_{m}\left(z, B_{x^{\prime} a^{\prime \prime}}^{y^{\prime} a^{\prime \prime}} .\right.
\end{aligned}
$$

Для других слагаемых $S_{i_{1} \ldots i_{m}}$ суммы $\Sigma_{m}$ мультииндекс $\left(i_{1}, \ldots, i_{m}\right)$ не совпадает с $(1$, $2, \ldots, m)$. Поэтому хотя бы одна из первых $m$ координат точек соответствующего бруca $\left(B_{a^{\prime} a^{\prime \prime}}^{d(\nu) x^{\prime \prime}}\right)_{i_{1} \ldots i_{m}}$ не зависит от $\nu$ и равна соответствующей координате точки $a$. Это дает возможность рассуждать, как в рассмотренном выше случае $k<m$, и получить $S_{i_{1} \ldots i_{m}}=0$. Из сказанного ясно, что

$$
\Sigma_{m}=S_{12 \ldots m}=V_{m}\left(z, B_{x^{\prime} a^{\prime \prime}}^{y^{\prime} a^{\prime \prime}}\right)
$$

Аналогично вычисляются и суммы $\Sigma_{m+1}, \ldots, \Sigma_{N}$. Имеющиеся отличия укажем на примере суммы $\Sigma_{m+1}$. При этом удобно кратко обозначать $k$-мерные брусы вида $\left(B_{a}^{d(\nu) x^{\prime \prime}}\right)_{i_{1} \ldots i_{k}}(k>m)$ с фиксированными индексами $i_{1}=1, i_{2}=2, \ldots, i_{m}=m$ как

$$
\left(B_{a}^{d(\nu) x^{\prime \prime}}\right)_{12 \ldots m i_{m+1} \ldots i_{k}} \equiv\left(B_{a}^{d(\nu) x^{\prime \prime}}\right)_{i_{m+1} \ldots i_{k}}^{*}
$$

Тогда для соответствующих слагаемых суммы $\Sigma_{m+1}$ получим по аналогии с (15):

$$
\begin{aligned}
S_{12 \ldots m i_{m+1}} & =\sum_{\nu_{1}=0}^{1} \cdots \sum_{\nu_{m}=0}^{1}(-1)^{\nu_{1}+\cdots+\nu_{m}} V_{m+1}\left[z,\left(B_{a^{\prime} a^{\prime \prime}}^{d(\nu) x^{\prime \prime}}\right)_{i_{m+1}}^{*}\right] \\
& =V_{m+1}\left[z,\left(B_{x^{\prime} a^{\prime \prime}}^{y^{\prime} x_{i_{m+1}}^{\prime \prime}}\right)^{*}\right.
\end{aligned}
$$


Если учесть, что для остальных индексов слагаемые $S_{i_{1} \ldots i_{m} i_{m+1}}$ суммы $\Sigma_{m+1}$ равны 0 , как и в случае суммы $\Sigma_{m}$, то

$$
\Sigma_{m+1}=\sum_{i_{m+1}=m+1}^{N} S_{12 \ldots m i_{m+1}}=\sum_{i_{m+1}=m+1}^{N} V_{m+1}\left[z,\left(B_{x^{\prime} a^{\prime \prime}}^{y^{\prime} x^{\prime \prime}}\right)_{i_{m+1}}^{*}\right]
$$

Продолжая такие же вычисления, можно найти выражения для всех сумм, входящих в (14). В итоге

$$
\begin{aligned}
& \sigma_{m}\left(\pi, B_{x^{\prime} x^{\prime \prime}}^{y^{\prime} x^{\prime \prime}}\right)=V_{m}\left(z, B_{x^{\prime} a^{\prime \prime}}^{y^{\prime} a^{\prime \prime}}\right)+\sum_{i_{m+1}=m+1}^{N} V_{m+1}\left[z,\left(B_{x^{\prime} a^{\prime \prime}}^{y^{\prime} x^{\prime \prime}}\right)_{i_{m+1}}^{*}\right] \\
& \quad+\sum_{i_{m+1}=m+1}^{N-1} \sum_{i_{m+2}=i_{m+1}+1}^{N} V_{m+2}\left[z,\left(B_{x^{\prime} a^{\prime \prime}}^{y^{\prime} x^{\prime \prime}}\right)_{i_{m+1} i_{m+2}}^{*}\right]+\cdots+V_{N}\left(z, B_{x^{\prime} a^{\prime \prime}}^{y^{\prime} x^{\prime \prime}}\right) \\
& =V_{m}\left(z, B_{x^{\prime} a^{\prime \prime}}^{y^{\prime} a^{\prime \prime}}\right)+\sum_{k=1}^{N-m} \sum_{i_{m+1}} \cdots \sum_{i_{m+k}} V_{m+k}\left[z,\left(B_{x^{\prime} a^{\prime \prime}}^{y^{\prime} x_{i}^{\prime \prime}}\right)_{i_{m+1} \ldots i_{m+k}}^{*}\right.
\end{aligned}
$$

Отсюда ясно, что $\sigma_{m}\left(\pi, B_{x^{\prime} x^{\prime \prime}}^{y^{\prime} x^{\prime \prime}}\right) \geqslant 0$. Отметим также, что для бруса вида $B_{x}^{y}=B_{x^{\prime} a^{\prime \prime}}^{y^{\prime} a^{\prime \prime}}$ равенство (16) имеет вид

$$
\sigma_{m}\left(\pi, B_{x}^{y}\right)=V_{m}\left(z, B_{x}^{y}\right)
$$

Продолжая доказательство, рассмотрим квазиобъем $\sigma_{m}\left(z, B_{x^{\prime} x^{\prime \prime}}^{y^{\prime} x^{\prime \prime}}\right)$. Согласно (1) и по теореме 2 (см. (8)) получим

$$
\begin{aligned}
\sigma_{m}\left(z, B_{x^{\prime} x^{\prime \prime}}^{y^{\prime} x^{\prime \prime}}\right) & =\sum_{\nu_{1}=0}^{1} \cdots \sum_{\nu_{m}=0}^{1}(-1)^{\nu_{1}+\cdots+\nu_{m}} z\left[y^{\prime}+\nu\left(x^{\prime}-y^{\prime}\right), x^{\prime \prime}\right] \\
& =\sum_{\nu_{1}=0}^{1} \cdots \sum_{\nu_{m}=0}^{1}(-1)^{\nu_{1}+\cdots+\nu_{m}}\left\{z\left[y^{\prime}+\nu\left(x^{\prime}-y^{\prime}\right), x^{\prime \prime}\right]-z\left(a^{\prime}, a^{\prime \prime}\right)\right\} \\
& =\sum_{\nu_{1}=0}^{1} \cdots \sum_{\nu_{m}=0}^{1}(-1)^{\nu_{1}+\cdots+\nu_{m}} \sum_{k=1}^{N} \sum_{\substack{i_{1} \\
1 \leqslant i_{1}<\cdots<i_{k} \leqslant N}} \cdots \sum_{i_{k}} \sigma_{k}\left[z,\left(B_{a^{\prime} a^{\prime \prime}}^{d(\nu) x^{\prime \prime}}\right)_{i_{1} \ldots i_{k}}\right] .
\end{aligned}
$$

Дальнейшие преобразования, проводимые как и при вычислении квазиобъема $\sigma_{m}(\pi$, $B_{x^{\prime} x^{\prime \prime}}^{y^{\prime} x^{\prime \prime}}$ с ф формальной заменой в $(13)-(16)$ вариаций $V_{k}(z, \cdot)$ на квазиобъемы $\sigma_{k}(z, \cdot)$, дают

$$
\begin{aligned}
\sigma_{m}\left(z, B_{x^{\prime} x^{\prime \prime}}^{y^{\prime} x^{\prime \prime}}\right)= & \sigma_{m}\left(z, B_{x^{\prime} a^{\prime \prime}}^{y^{\prime} a^{\prime \prime}}\right) \\
& +\sum_{k=1}^{N-m} \sum_{\substack{i_{m+1} \\
m+1 \leqslant i_{m+1}<\cdots<i_{m+k} \leqslant N}} \cdots \sum_{\substack{i_{m+k} \\
m-m}} \sigma_{m+k}\left[z,\left(B_{x^{\prime} a^{\prime \prime}}^{y^{\prime} x^{\prime \prime}}\right)_{i_{m+1} \ldots i_{m+k}}^{*}\right] .
\end{aligned}
$$


Учитьвая равенство $\mu(x)=\pi(x)-z(x)$ и линейность квазиобъема по производящей функции (см. (1)), из формул (16), (17) получим

$$
\begin{aligned}
\sigma_{m}\left(\mu, B_{x^{\prime} x^{\prime \prime}}^{y^{\prime} x^{\prime \prime}}\right)= & \sigma_{m}\left(\pi, B_{x^{\prime} x^{\prime \prime}}^{y^{\prime} x^{\prime \prime}}\right)-\sigma_{m}\left(z, B_{x^{\prime} x^{\prime \prime}}^{y^{\prime} x^{\prime \prime}}\right)=\left[V_{m}\left(z, B_{x^{\prime} a^{\prime \prime}}^{y^{\prime} a^{\prime \prime}}\right)-\sigma_{m}\left(z, B_{x^{\prime} a^{\prime \prime}}^{y^{\prime} a^{\prime \prime}}\right)\right] \\
& +\sum_{k=1}^{N-m} \sum_{\substack{i_{m+1} \\
m+1 \leqslant i_{m+1}<\cdots<i_{m+k} \leqslant N}} \cdots \sum_{i_{m+k}}\left\{V_{m+k}\left[z,\left(B_{x^{\prime} a^{\prime \prime}}^{y^{\prime} x^{\prime \prime}}\right)_{i_{m+1} \ldots i_{m+k}}^{*}\right]\right. \\
& \left.-\sigma_{m+k}\left[z,\left(B_{x^{\prime} a^{\prime \prime}}^{y^{\prime} x^{\prime \prime}}\right)_{i_{m+1} \ldots i_{m+k}}^{*}\right]\right\} \geqslant 0 .
\end{aligned}
$$

Последнее неравенство следует из определения вариаций $V_{m}, \ldots, V_{N}$.

Аналогичные выкладки и рассуждения можно провести и для других $m$-мерных брусов $B_{x}^{y} \subset B_{a}^{b}$. Таким образом, квазиобъемы $\sigma_{m}\left(\pi, B_{x}^{y}\right)$ и $\sigma_{m}\left(\mu, B_{x}^{y}\right)$, порождаемые функциями $\pi$ и $\mu$, неотрицательны для любых $m$-мерных брусов $B_{x}^{y}$, причем это верно для каждого $m, 1 \leqslant m \leqslant N$. Поэтому согласно определению $2 \pi(x), \mu(x) \in \mathscr{M}(B)$.

СлЕДСТВИЕ 3. Для того чтобы функиия $z(x)$ имела конечную полную вариацию в $B$, необходимо и достаточно ее представление в виде $z(x)=\widetilde{\pi}(x)-\widetilde{\mu}(x)$, где $\widetilde{\pi}(x), \widetilde{\mu}(x) \in \mathscr{M}(B)$.

В работах [1]-[3], [6], [15] изучался класс Харди $\mathscr{V}_{H}(B)$ функций с ограниченными вариациями. Он состоит из функций $z(x)$, заданньх в $B \subset \mathbb{R}^{N}$, для которых при любом $m(1 \leqslant m \leqslant N) m$-мерные вариации функции $z$ по произвольному $m$-мерному координатному сечению бруса $B$ конечны. В [6], [15] указано, что необходимым и достаточным условием принадлежности $z(x) \in \mathscr{V}_{H}(B)$ является представимость функции в виде $z(x)=\widetilde{\pi}(x)-\widetilde{\mu}(x)$, где $\widetilde{\pi}(x), \widetilde{\mu}(x)$ - полностью монотонные функции. Поэтому из следствия 3 вытекает

СЛЕДСТВИЕ 4. Классы $\mathscr{V}(B) u \mathscr{V}_{H}(B)$ совпадают.

Отсюда и из следствия 1 получается

СлЕДСТВИЕ 5. Если $z \in \mathscr{V}(B)$, то для любьх $x, y \in B, x \leqslant y$, полная вариаиия $V\left(z, B_{x}^{y}\right)$ конечна $u|z(x)-z(y)| \leqslant V\left(z, B_{x}^{y}\right)$.

4. Принцип выбора Хелли в $N$-мерном случае. Функции класса Харди $\mathscr{V}_{H}(B)$ достаточно хорошо изучены, и их свойства, дополненные результатами следствий $1,2,5$ и теоремы 3 , в основном аналогичны свойствам функций одной переменной с ограниченной вариацией. Исключение составляет принцип выбора Хелли. В литературе известен следующий вариант этого принципа при $N>1$ (см. [7, с. 331], [8, с. 311]).

Лемма 2. Если $\left\{z_{n}(x)\right\} \subset \mathscr{M}(B) u\left|z_{n}(x)\right| \leqslant K=$ const для любого $x \in B$, то найдутся подпоследовательность $\left\{z_{n_{k}}(x)\right\} \subset\left\{z_{n}(x)\right\}$ и функиия $z(x) \in \mathscr{M}(B)$ такие, что $z_{n_{k}}(x)$ сходится $\kappa z(x)$ для всех $x$ - точек непрерывности функиии $z(x)$ (т.е. "сходится в существенном", см. [7, с. 330]).

Таким образом, вариант принципа выбора Хелли при $N>1$ соответствует не выбору поточечно сходящейся подпоследовательности, а выбору подпоследовательности, сходящейся "в существенном". Тем не менее, как будет показано ниже, принцип выбора Хелли при $N>1$ справедлив и для поточечной сходимости в классе функций $\mathscr{V}(B)$. 
Лемма 3. Пусть $\{z(x)\}$ - семейство, содержащее бесконечное число функций $z(x) \in \mathscr{M}(B)$, ограниченных в $B \subset \mathbb{R}^{N}$ одной и той жсе константой: $|z(x)| \leqslant K$. Тогда из $\{z(x)\}$ мохно выбрать последовательность $\left\{z_{n}(x)\right\}$, поточечно сходящуюся в $B$ к функиии из класса $\mathscr{M}(B)$.

ДокАЗАТЕЛЬСТвО проведем по методу математической индукции. При $N=1$ лемма содержит известное утверждение теории монотонных функций (см. [5, с. 208]). Пусть лемма верна для функций $(N-1)$-й переменной. Установим ее справедливость для функций $N$ переменных.

По лемме 2 из семейства $\{z(x)\}$ можно выбрать последовательность $\left\{z_{n}(x)\right\} \subset \mathscr{M}(B)$, сходящуюся к некоторой функции $z_{0}(x) \in \mathscr{M}(B)$ в ее точках непрерывности. При этом по лемме $1 z_{0}(x)$ непрерывна в $B$ всюду за исключением, быть может, точек, лежащих на не более чем счетном множестве координатных гиперплоскостей размерности $N-1$. Обозначим эти гиперплоскости через $Q_{j}(j=1,2, \ldots)$, а их объединение - через $Q$. Тогда $z_{n}(x) \rightarrow z_{0}(x)$ при любом $x \in B \backslash Q$.

Введем для каждого $j$ последовательность функций $(N-1)$-й переменной: $z_{n}^{(j)}(x)=$ $\left\{z_{n}(x): x \in B \cap Q_{j}\right\}(n=1,2, \ldots)$. При фиксированном $j$ каждая из функций $z_{n}^{(j)}(x)$ принадлежит классу $\mathscr{M}\left(B \cap Q_{j}\right)$. Все эти функции ограничены константой $K$. Поэтому согласно индукционному предположению из $\left\{z_{n}^{(1)}(x)\right\}$ можно извлечь поточечно сходящуюся на $B \cap Q_{1}$ подпоследовательность $\left\{z_{p_{1}}^{(1)}(x)\right\}$. Здесь $\left\{p_{1}\right\}$ - возрастающая бесконечная подпоследовательность индексов: $\left\{p_{1}\right\} \subset\{n\}$. Соответствующая подпоследовательность функций $N$ переменных $\left\{z_{p_{1}}(x)\right\},\left\{z_{p_{1}}(x)\right\} \subset\left\{z_{n}(x)\right\}$, будет сходиться не только на $B \backslash Q$, но и на $B \cap Q_{1}$. Аналогично, из подпоследовательности $\left\{z_{p_{1}}^{(2)}(x)\right\}$, $\left\{z_{p_{1}}^{(2)}(x)\right\} \subset\left\{z_{n}^{(2)}(x)\right\}$, функций $(N-1)$-го переменного можно выбрать сходящуюся на $B \cap Q_{2}$ подпоследовательность $\left\{z_{p_{2}}^{(2)}(x)\right\},\left\{p_{2}\right\} \subset\left\{p_{1}\right\}$. Тогда подпоследовательность $\left\{z_{p_{2}}(x)\right\},\left\{z_{p_{2}}(x)\right\} \subset\left\{z_{p_{1}}(x)\right\} \subset\left\{z_{n}(x)\right\}$, функций $N$ переменных будет сходиться на $B \backslash Q, B \cap Q_{1}$ и $B \cap Q_{2}$. Продолжая этот процесс, получим цепочку подпоследовательностей функций $N$ переменных: $\cdots \subset\left\{z_{p_{j+1}}(x)\right\} \subset\left\{z_{p_{j}}(x)\right\} \subset \cdots \subset\left\{z_{p_{1}}(x)\right\} \subset\left\{z_{n}(x)\right\}$, $j=1,2, \ldots$, причем $\left\{z_{p_{j}}(x)\right\}$ сходится на $B \backslash Q, B \cap Q_{1}, \ldots, B \cap Q_{j}$. Построим теперь “диагональную” подпоследовательность $\left\{z_{i}(x)\right\}_{i=1}^{\infty},\left\{z_{i}(x)\right\} \subset\left\{z_{n}(x)\right\}$, в которой $z_{i}(x)$ есть $i$-й член подпоследовательности $\left\{z_{p_{i}}(x)\right\}$. Она сходится на $B \backslash Q$ и на любом множестве $B \cap Q_{j}$. Действительно, для каждого фиксированного $j$ последовательность $\left\{z_{i}(x)\right\}_{i=1}^{\infty}$ при $i \geqslant j$ есть подпоследовательность, выбранная из $\left\{z_{p_{j}}(x)\right\}$, и поэтому она сходится на $B \cap Q_{j}$. Таким образом, $\left\{z_{i}(x)\right\}$ сходится всюду на $B$.

Обозначим предельную функцию для $\left\{z_{i}(x)\right\}$ как $z^{*}(x)$. Докажем, что $z^{*}(x) \in \mathscr{M}(B)$. Для этого возьмем произвольньй брус $B_{x}^{y}, B_{x}^{y} \subset B, x \leqslant y$, любой размерности $m$, $1 \leqslant m \leqslant N$, и вычислим по формуле типа (1) квазиобъемы $\sigma_{m}\left(z_{i}, B_{x}^{y}\right), \sigma_{m}\left(z^{*}, B_{x}^{y}\right)$, которые порождаются функциями $z_{i}(x)$ и $z^{*}(x)$ соответственно. Так как величины этих квазиобъемов выражаются в виде линейной комбинации значений производящей функции в вершинах бруса, то из установленной поточечной сходимости $z_{i}(x) \rightarrow z^{*}(x)(i \rightarrow \infty)$ в $B$ следует, что $\sigma_{m}\left(z_{i}, B_{x}^{y}\right) \rightarrow \sigma_{m}\left(z^{*}, B_{x}^{y}\right)$. Это вместе с неотрищательностью величин $\sigma_{m}\left(z_{i}, B_{x}^{y}\right)$ для функций $z_{i}(x) \in \mathscr{M}(B)$ доказьвает, что $\sigma_{m}\left(z^{*}, B_{x}^{y}\right) \geqslant 0$. Тогда по определению $2 z^{*}(x) \in \mathscr{M}(B)$. Лемма доказана.

Из леммы 3 , используя разложение функций $z(x) \in \mathscr{V}(B)$, данное в теореме 3 , формулу (12) и рассуждая дословно так же, как и в случае $N=1$ (см. [5, с. 209]), можно 
получить аналог принципа выбора Хелли для функций $N$ переменных с ограниченной полной вариацией.

ТЕОрема 4. Если для семейства функиий $\{z(x)\} \subset \mathscr{V}(B)$, содержсащего бесконечное число әлементов, выполнено условие $\|z\|_{V} \leqslant K=$ const, то найдется последовательность функиий, принадлежащих этому семейству, которая сходится поточечно в $B$ к функиии из $\mathcal{V}(B)$.

\section{СПИСОК ЦИТИРОВАННОЙ ЛИТЕРАТУРЫ}

[1] Hahn H. Theorie der reellen Funktionen. I. Berlin: Springer, 1921.

[2] Hobson E. W. The theory of functions of a real variable and the theory of Fourier's series. 3rd ed. V. 1. Cambridge: Cambridge Univ. Press, 1927.

[3] Clarkson J.A., Adams C. R. On the definition of bounded variation for functions of two variables // Trans. Amer. Math. Soc. 1933. V. 35. № 4. P. 824-854.

[4] Витушкин А. Г. О многомерных вариациях. М.: Гостехиздат, 1955.

[5] Натансон И. П. Теория функций вещественной переменной. М.: Наука, 1974.

[6] Hardy G. H. On double Fourier series, and especially those which represent the double zetafunctions with real and incommensurable parameters // Quart. J. Math. Oxford. 1905. V. 37. P. 53-79.

[7] Бохнер С. Лекции об интегралах Фурье (с добавлением автора о монотонных функциях, интегралах Стилтьеса и гармоническом анализе). М.: Физматгиз, 1962.

[8] Биллингсли П. Сходимость вероятностных мер. М.: Наука, 1977.

[9] Иванов Л. Д. Вариации множеств и функций. М.: Наука, 1975.

[10] Тихонов А. Н., Леонов А. С., Ягола А. Г. Нелинейные некорректные задачи. М.: Наука, 1995.

[11] Камке Э. Интеграл Лебега-Стилтьеса. М.: Физматгиз, 1959.

[12] Шилов Г. Е., Гуревич Б. Л. Интеграл, мера, производная. М.: Наука, 1967.

[13] Канторович Л.В., Акилов Г. П. Функциональный анализ. М.: Наука, 1977.

[14] Young W. H., Young G. C. On the discontinuities of monotone functions of several variables // Proc. London Math. Soc. (2). 1924. V. 22. P. 124-142.

[15] Adams C.R., Clarkson J.A. Properties of functions $f(x, y)$ of bounded variation // Trans. Amer. Math. Soc. 1934. V. 36. № 4. P. 711-730. 